\title{
The Framework of Novel K-means Embedded Cloud Computing Platform for Real-time Unmanned Aerial Vehicle (UAV) Remote Sensing Images Processing
}

\author{
Lin, Feng-Cheng ${ }^{1}$ \\ GIS Research Center, Feng Chia University \\ No. 100, Wenhwa Rd., Seatwen, Taichung, Taiwan \\ E-mail:francis@gis.tw \\ Wang, Chun-Ju \\ Department of Urban Planning and Spatial Information, Feng Chia University \\ No. 100, Wenhwa Rd., Seatwen, Taichung, Taiwan \\ E-mail: st_ruby@gis.tw
}

\author{
Chung, Lan-Kun; Ho, Chung-Han; Chou, Tien-Yin \\ GIS Research Center, Feng Chia University \\ No. 100, Wenhwa Rd., Seatwen, Taichung, Taiwan \\ E-mail:peter@gis.tw;john@gis.tw; jimmy@gis.tw
}

Due to the rapid development of remote sensing (RS) technology in recent years, high-quality images shot by Unmanned Aerial Vehicle (UAV) have become so widespread in the usage of environmental observation and record. Then further RS image processing works can be done by standalone software, ex. ENVI, but there are two bottlenecks in processing a large amount of RS images: limitation of computation and capacity of storage. Therefore, researchers have developed many kinds of variants in parallel algorithms, and most of them are implemented by using MPI or MapReduce. In this paper, we propose and implement a framework of novel $K$ means algorithm based on MapReduce architectures. The observer on the ground can lay their finger to decide central points (centroids) of real-time images shot by UAV in person by tablet computer. The clustered result is completed by our proposed cloud computing environment and appeared by color blocks in front of tablet computer within the acceptable time. From our experiment, human intervention (man-made centroid) can get faster convergence better than random centroid selection. Finally, this service can be used in our UAV business and become software of a service (SaaS) in hadoop environment.

The International Symposium on Grids and Clouds (ISGC) 2013

March 17-22, 2013

Academia Sinica, Taipei, Taiwan

1 Speaker 


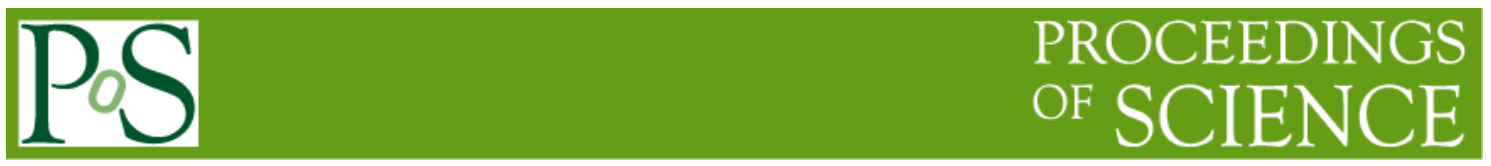

\section{Introduction}

To collect specified aerial images, GIS Research Center of Feng Chia University (GIS.FCU) developed Unmanned Aerial Vehicle (UAV) that integrates aerial engineering and electronics, wireless transmission, image post-processing, and GPS technique's coordination with OGC-SWE compatible software for comprehensive crossplatform environmental investigation, such as debris-flow monitoring, river monitoring, urban planning or planning for agriculture. From the "sensor web" in wikipedia (http://en.wikipedia.org/wiki/Sensor_web), Open Geospatial Consortium's Sensor Web Enablement (OGC-SWE) defines service interfaces which enable an interoperable usage of sensor resources by enabling their discovery, access, tasking, as well as eventing and alerting. The appearance of our UAV (named for AS-4) is shown in Fig. 1. Since AS-4 is configured with the related modules given in Fig. 2, UAV can shoot high-resolution picture or HD movie with a camera in sky, and operator can control UAV by real-time monitoring software installed in the personal computer on the ground in Fig. 3. When a disaster occurs, rescuers can classify images by our cloud environment and help them to know the distribution of disaster immediately.

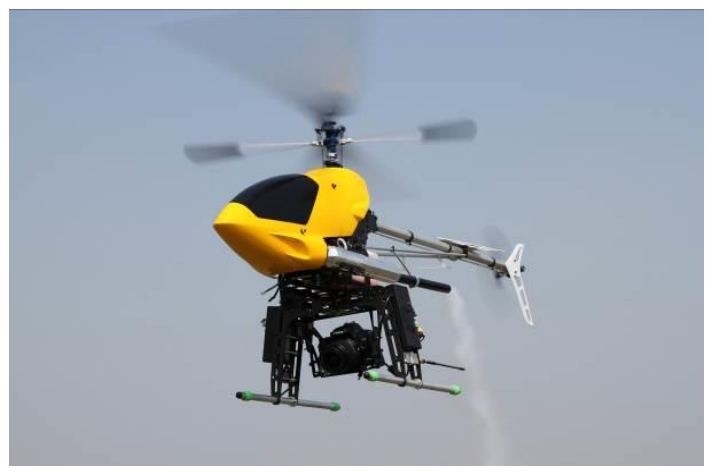

Fig. 1. Unmanned Aerial Vehicle (named for AS-4) of GIS.FCU.

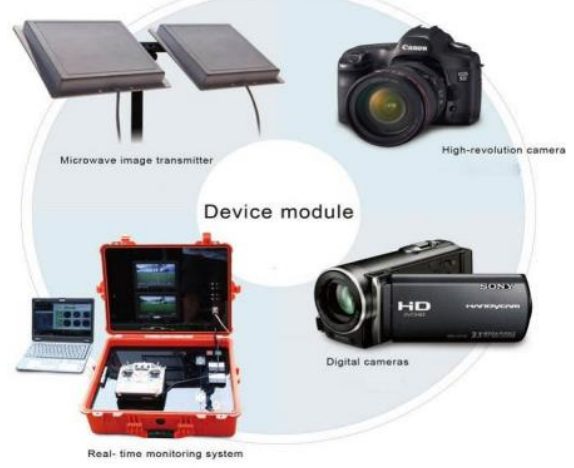

Fig. 2. Relevant modules of AS-4. 

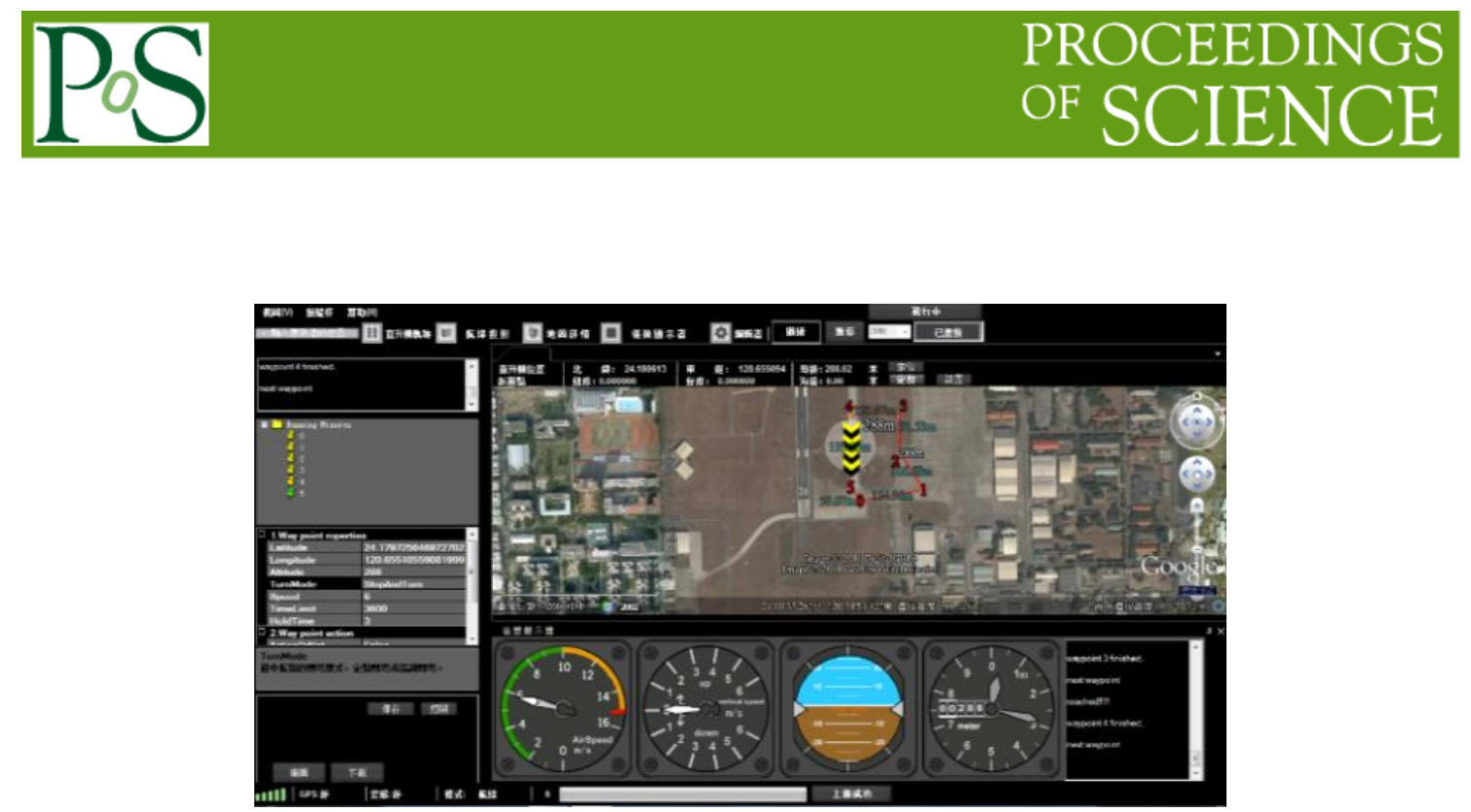

Fig. 3. Automatic flying system in ground control station.

Nowadays, camera of UAV captures images at a higher resolution that they will occupy more hard disk space, and image's processing will cost more time; therefore, we need to have a well-designed platform to store remote sensing images and process images effectively in a distributed environment. We have developed a research of managing the massive remote sensing images, used the distributed computation technology on several different runtimes cloud environments in this paper, and the efficiency improved obviously.

MapReduce proposed by Google has been widely used for large-scale data analysis in the cloud. This kind of programming model simplified data processing on large clusters. In the past six years, Hadoop project realized the mass data storage (HDFS)/analysis (MapReduce). These existed literatures are focus on computational ability using MapReduce. However, we focus on a total solution in business usage combining with our UAV product.

Therefore, the main contributions of this paper are to propose a framework and then implement a system that can store and process massive remote sensing images on a cloud computing platform. Based on above concepts, we propose and implement a framework of novel $K$-means algorithm under two kinds of cloud environments. After shooting by UAV, user can select the initial points (man-made centroids) of $K$-means algorithm on the tablet computer to replace initial centroids by random, and it will calculate cluster centroids repeatedly until this algorithm converges (the centroid of each cluster will not change).

The remainder of this paper was organized as following. The section II describes the concept of $K$-means and the calculation of $K$-means under the cloud environments. The section III is the design and implementation of the system architecture; it describes the cloud platform of processing the remote sensing image and the front-end of the 


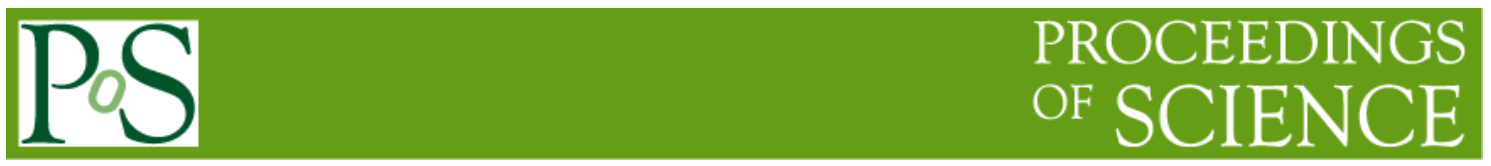

tablet computer. Then, we compare the performance between three-machines and VMmanagement (Hyper-V) cloud computing in section IV. Finally, section V concludes this paper.

\section{Research Methods}

There are two main image classification methods: Supervised Classification and Unsupervised Classification. In this paper, we apply unsupervised classification by $K$ means algorithms that have highly flexible, efficient, and easy advantages.

$K$-means was first used by MacQueen in 1967 [1]. It is one of the most extensive cluster analyses so far. We proposed a new concept that user could select $K$ initial centroids themselves, and system calculated the distance between each pixel and centroids; each pixel will be assigned to their own cluster where the distance from the pixel to centroid of own cluster is minimized. After that, it will redefine the center of each cluster until the termination condition is met [5]. In this paper, we propose this improved algorithm where initial centroids are man-made instead of random ones.

MapReduce is the most crucial technology in Hadoop-based cloud environment; it can process images in distributed environment. One image will be splitted several files (RGB value of each pixel in a given image) based on the block size, and system will save them on multiple data nodes. Map function computes what cluster each pixel belongs to and generates an input data of key value to reflect an output key value. Reduce function gathers all points and classifies them into different clusters depending on their belongings. We implement above concept in $K$-means algorithms to complete images clustering. Finally, the whole picture of proposed approach is shown in Fig. 4. 


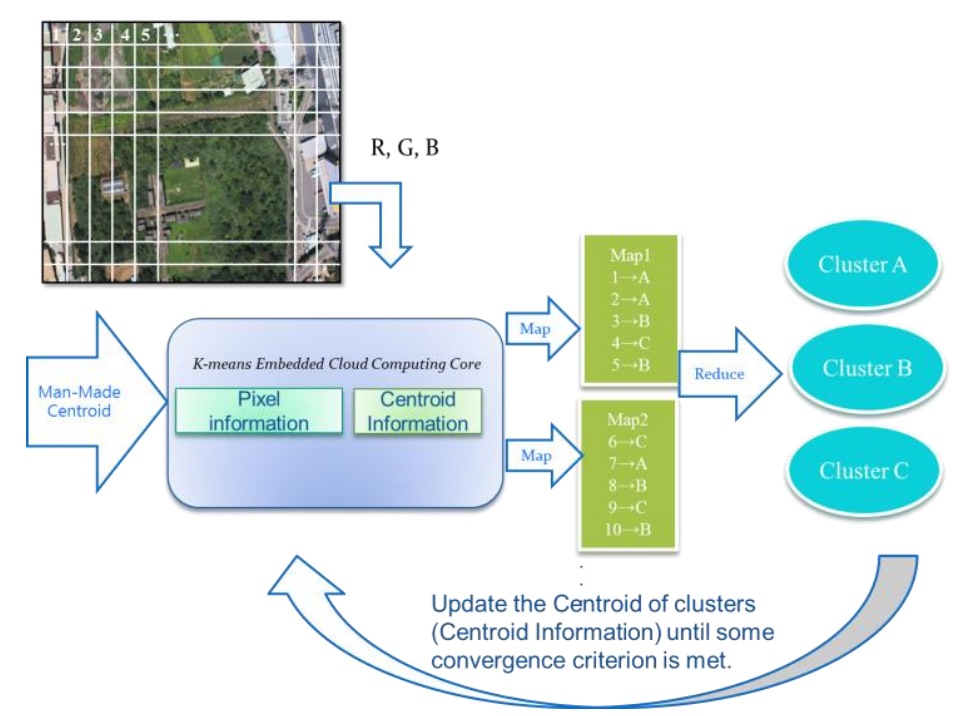

Fig. 4. MapReduce-based novel $K$-means architecture.

\section{System Architecture}

This paper implements Remote Sensing Image Classification in tablet computer based on cloud technology, and corresponding system architecture was presented in Fig. 5 that includes service entrance, data processing, and Hadoop environment. We will introduce each module in the following.

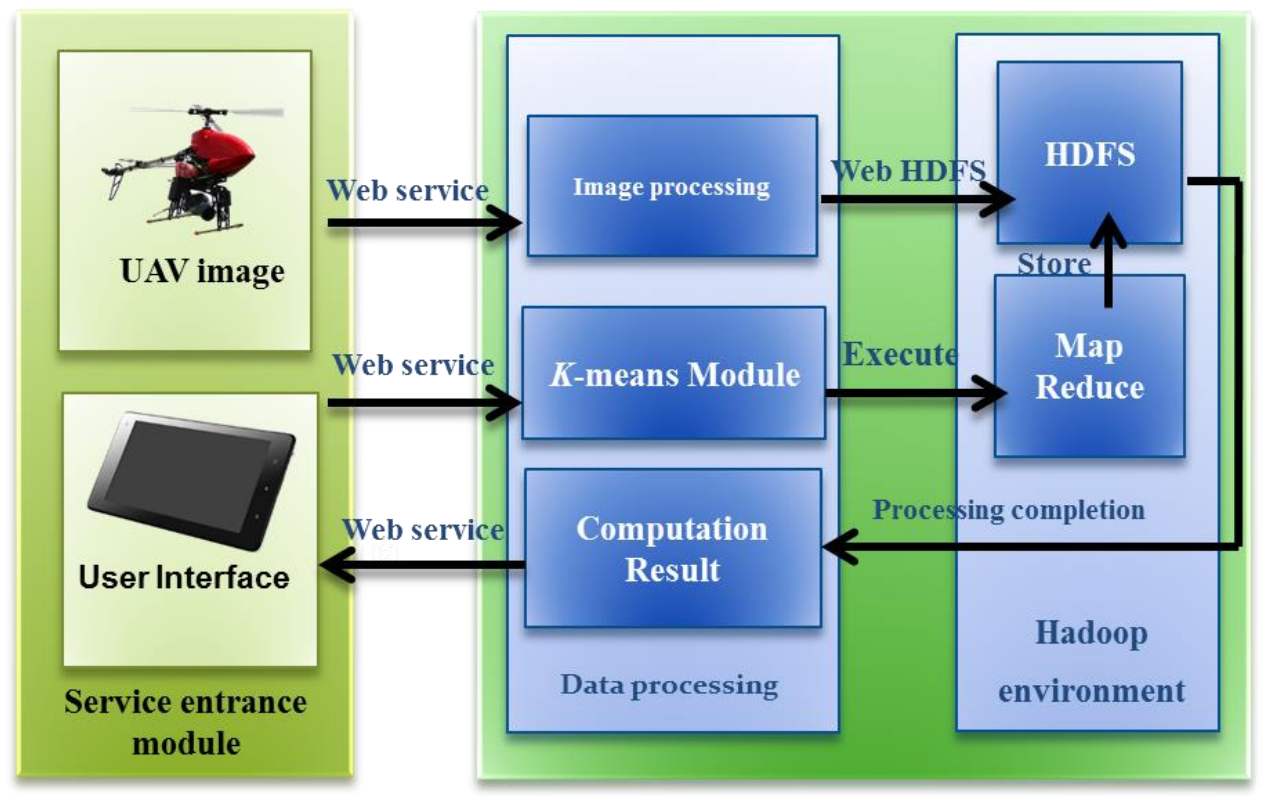

Fig. 5. The Framework of Novel $K$-means Embedded Cloud Computing Platform.

A. Service entrance module 
After rescuers shot immediate images with UAV, the platform preprocess images through web service, and store them in HDFS systems. Therefore, users can identify images immediately by tablet computer.

B. Data processing module

Data processing is a communicating interface to connect Service entrance module and Hadoop Environment. When users input remote sensing image of UAV and receives requests from users by service entrance module, it assigns works to data processing module that includes three processing modules:

(a) Images preprocessing module

RGB value of UAV's image is analyzed by images preprocessing module, and system keeps images in HDFS system by WebHDFS.

(b) $K$-means Module

After users obtain RGB value of images, they use $K$-means Module to process image classification in MapReduce framework [3, 4]. It can identify images faster and store them in HDFS by Web HDFS in Hadoop environment.

(c) Computation Result

We use HDFS system to store computational result of $K$-means Module [2]. This result is exhibited visually by calling Computation Result web service shown in pad.

C. Hadoop environment

Hadoop is an open-source software framework that comes from Apache Software Foundation [4] where HDFS and MapReduce are two important cores. We can store UAV images and detailed information in HDFS system, and proposed platform deals with images by MapReduce framework

\section{Results of the experiments}

In this chapter, we implement UAV Remote Sensing Images Processing (UAVRSIP) in Asus transformer pad. First, the home of UAV-RSIP is shown in Fig. 6. Second, we can choose one image that will be sent and analyzed in the cloud as Fig. 7(a) and Fig. 7(b) from SD card of Camera hanging on the UAV. To test real case, we took pictures and selected one by UAV in Longtan of Taoyuan, Taiwan. The selected image is $11,197,444$ pixels and 6.79 MB. Third, user selects directly $K$ original centroids in 


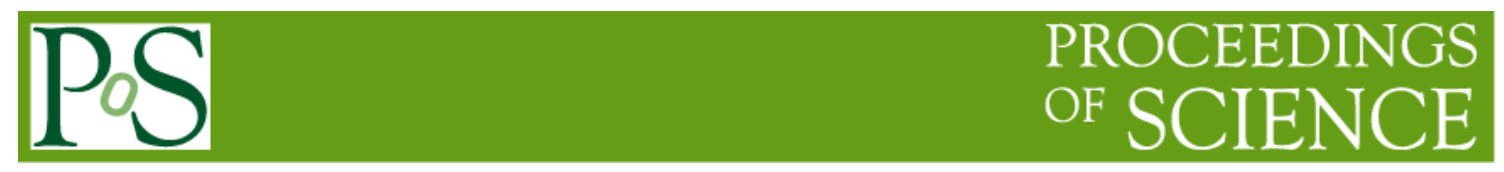

UAV images in Fig. 8(a), and then this module will call the module of $K$-means to do process in cloud environment to obtain a clustered image in pad.

When a user clicks several points (man-made centroids) on pad, app of pad will keep values of XY coordinates and RGB of these centroids and send them into the cloud. To show the results, we set up $K=4 \& K=5$ and get the results of experiments in Fig. 8 (b) and Fig. 8(c) respectively. There are many pixels keep the same information (the same RGB value in Fig. 9(a)); therefore, we can just hold unduplicated RGB information (eliminate duplication) by a new record form as Fig. 9(b) to speed the convergence rate of $K$-maens algorithm. By the way, we may not know what kinds of cloud environment International Data Corporation (IDC) provided, so we also built two kinds of cloud environments in Fig. 10 and ran our experiments in Fig. 11.

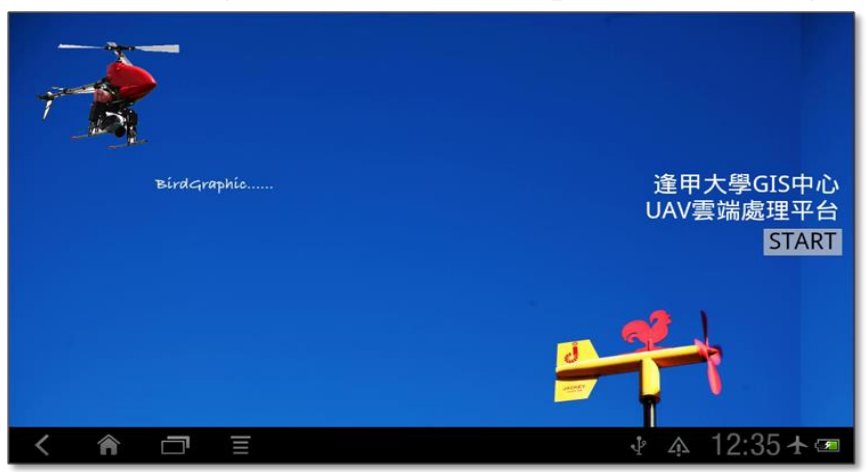

Fig. 6. The home of UAV-RSIP.

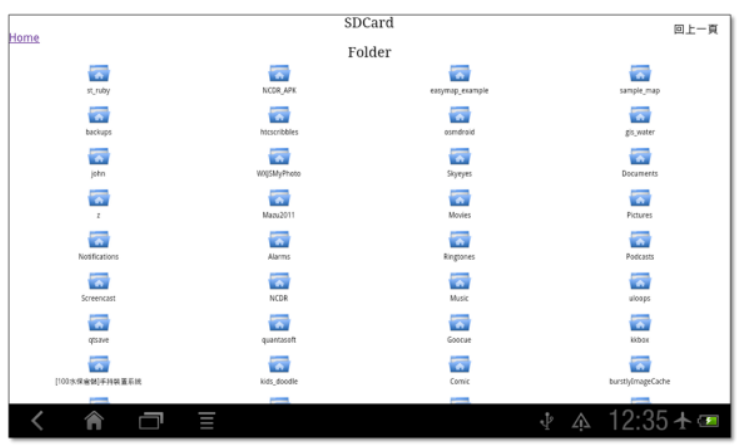

(a) The architecture of SDCard in pad Fig. 7. Select one image among all images in SD card form camera in UAV.

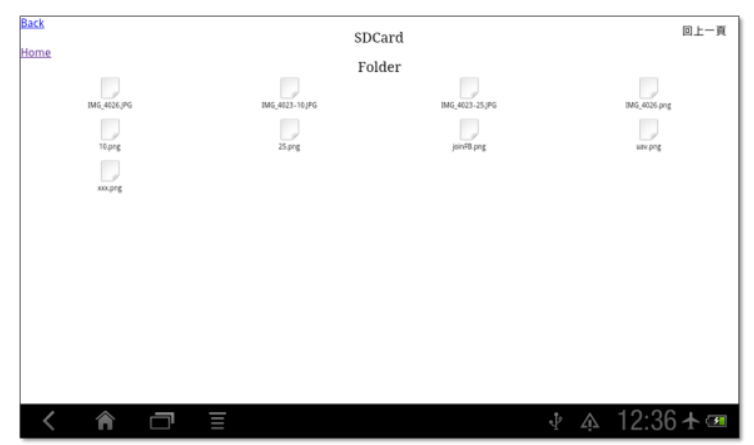

(b) Select one image from SDCard 

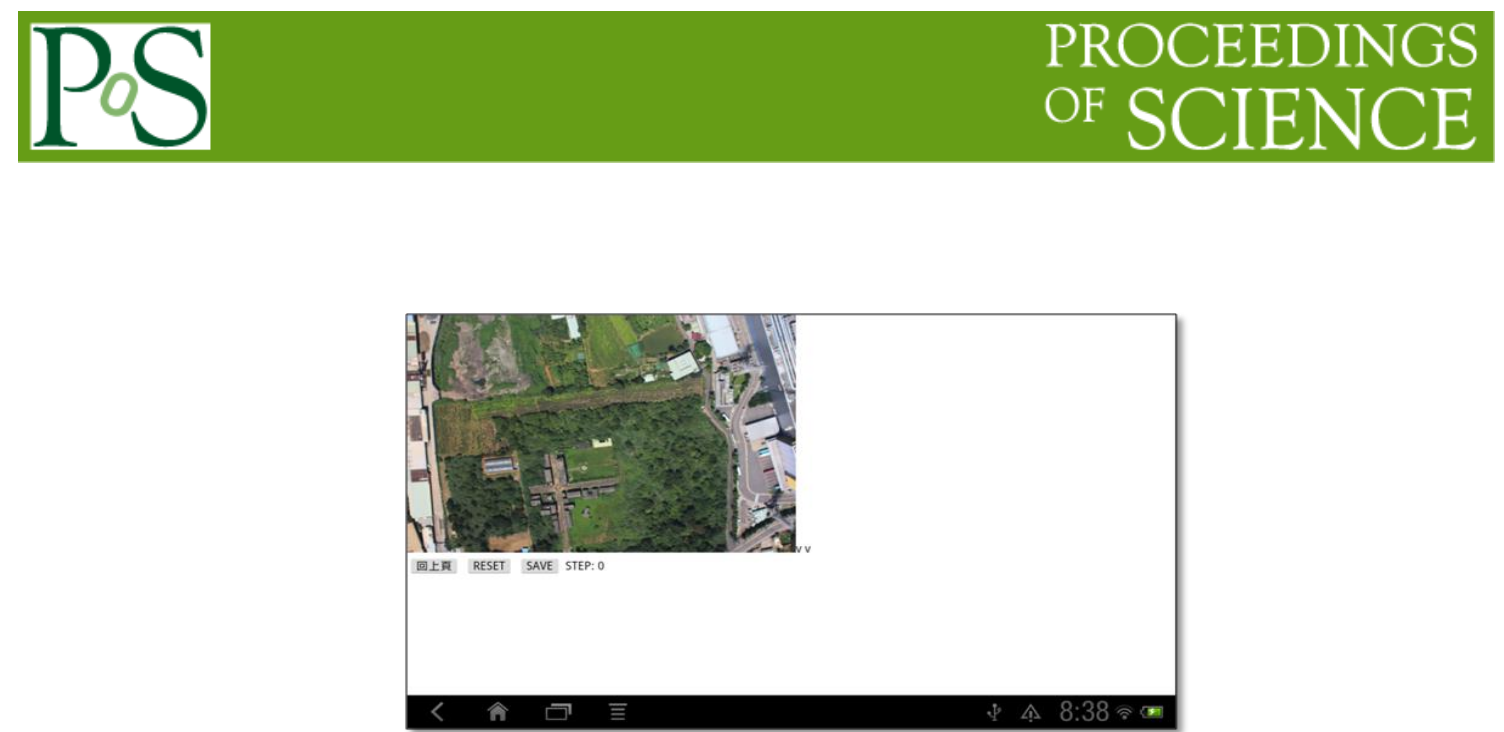

(a) The selected image after completing the step in Fig. 7.
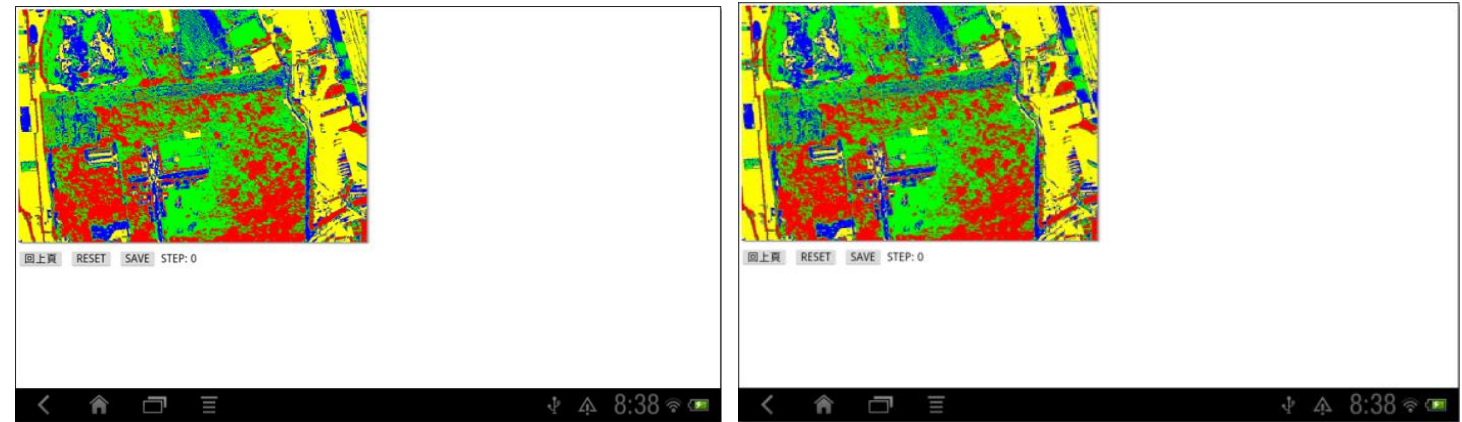

(b) The clustered result $(K=4)$

(c) The clustered result $(K=5)$

Fig. 8. The results of the experiment.

(a) Duplicate information
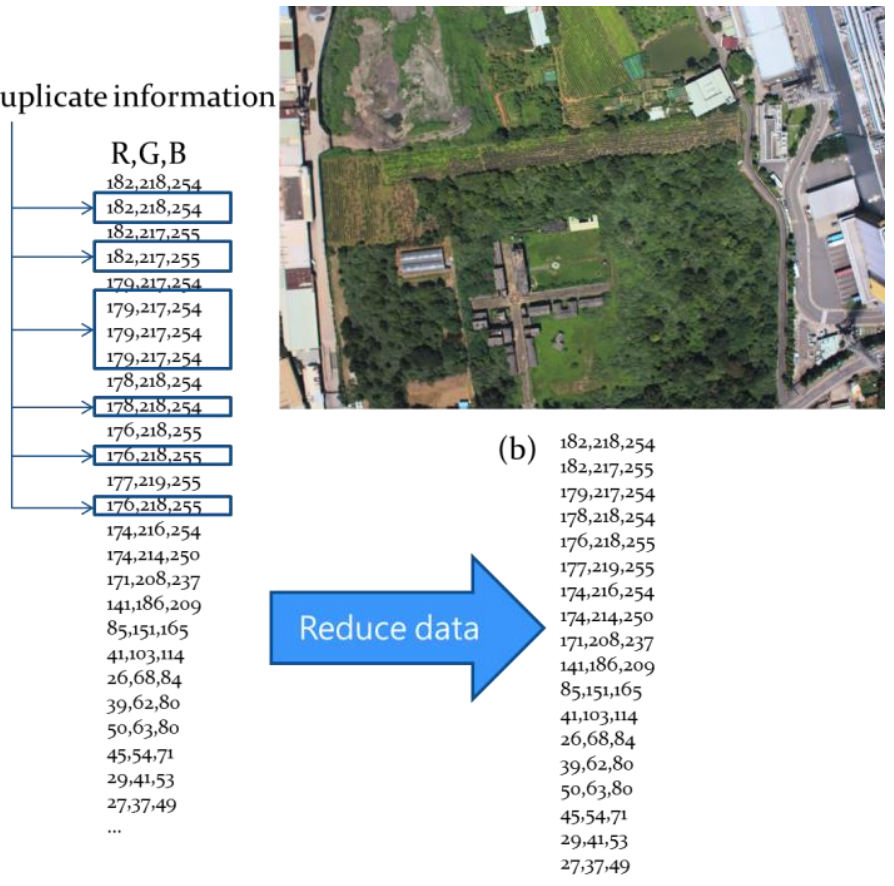

Fig. 9. The ideas of reducing duplicate information in one image. 
No. 1

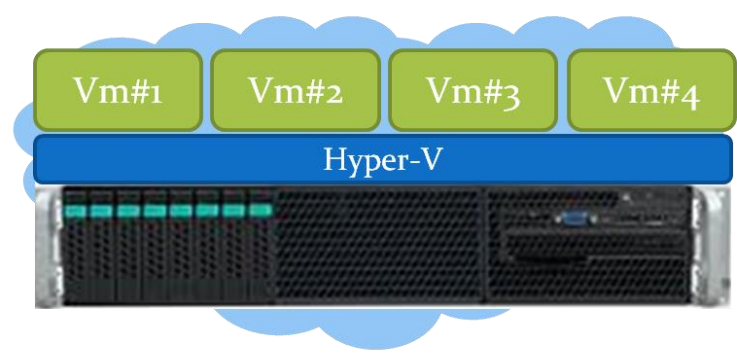

No. 2
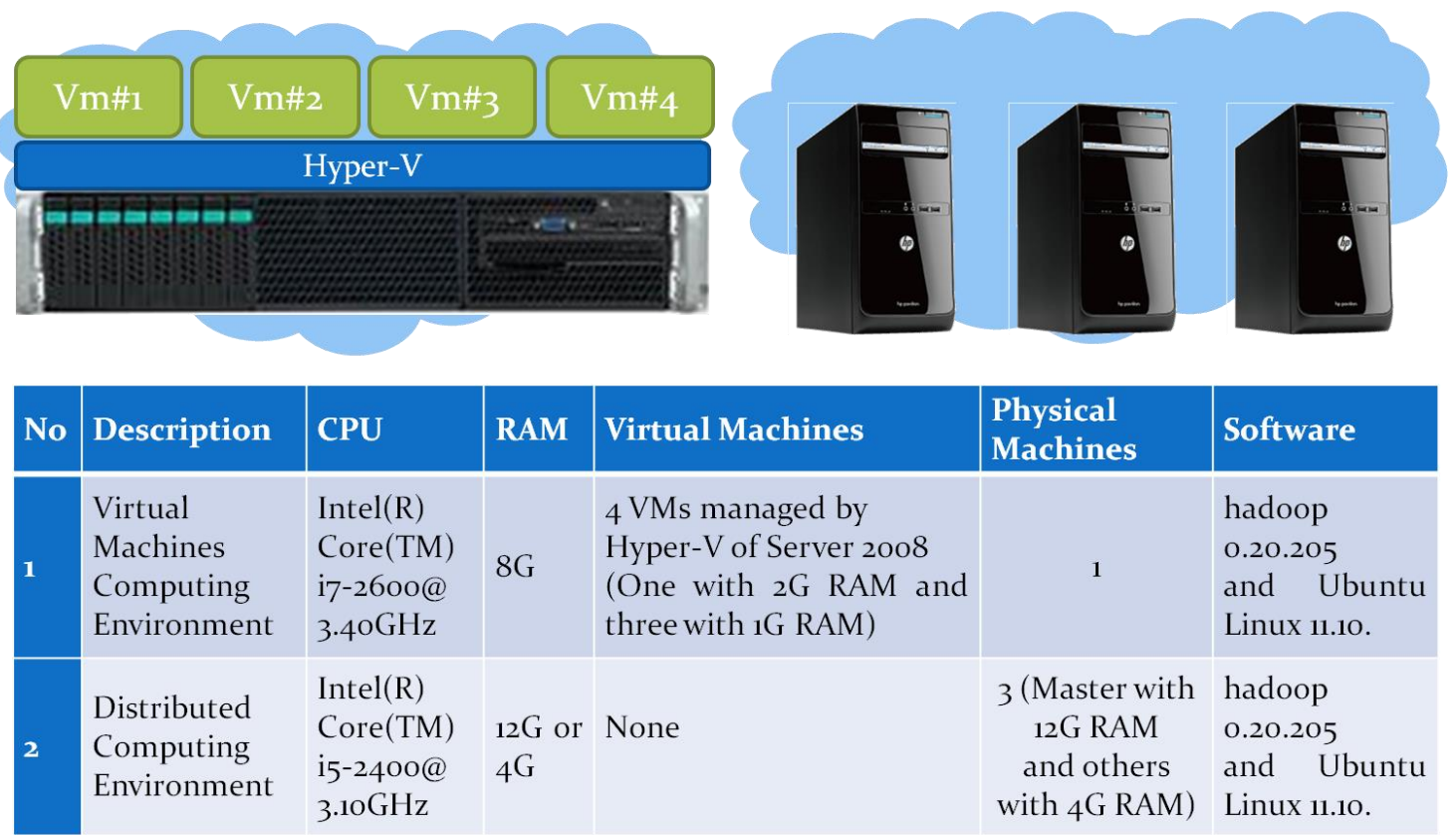

\begin{tabular}{|c|c|c|c|}
\hline Description & CPU & RAM & Virtua \\
\hline $\begin{array}{l}\text { Virtual } \\
\text { Machines } \\
\text { Computing } \\
\text { Environment }\end{array}$ & $\begin{array}{l}\text { Intel(R) } \\
\text { Core(TM) } \\
\text { i7-260o@ } \\
\text { 3.40GHz }\end{array}$ & $8 G$ & $\begin{array}{l}4 \text { VMs } \\
\text { Hyper- } \\
\text { (One } \\
\text { three }\end{array}$ \\
\hline $\begin{array}{l}\text { Distributed } \\
\text { Computing } \\
\text { Environment }\end{array}$ & $\begin{array}{l}\text { Intel(R) } \\
\text { Core(TM) } \\
\text { i5-2400@ } \\
3.10 G H z\end{array}$ & $\begin{array}{l}{ }_{12} G \text { or } \\
{ }_{4} G\end{array}$ & None \\
\hline
\end{tabular}

Fig. 10. Setup Hadoop in two kinds of environments.

\begin{tabular}{|c|c|c|c|c|}
\hline \multicolumn{3}{|c|}{$K=5$} & \multicolumn{2}{|c|}{$K=10$} \\
\hline \multicolumn{5}{|c|}{ Original Data $(11,197,444$ points $)$} \\
\hline Average time $($ Run $=10)$ & Map=1 & Map=12 & Map=1 & Map $=12$ \\
\hline Random Centroid & $396.30 \mathrm{Sec}$ & $354.79 \mathrm{Sec}$ & $515.79 \mathrm{Sec}$ & $396.01 \mathrm{Sec}$ \\
\hline Man-Made Centroid & $311.81 \mathrm{Sec}$ & $325.76 \mathrm{Sec}$ & $388.85 \mathrm{Sec}$ & $332.64 \mathrm{Sec}$ \\
\hline \multicolumn{5}{|c|}{ Reduced Data (165,707 points) } \\
\hline & Map=1 & Map=2 & Map $=1$ & Map=2 \\
\hline Random Centroid & $375.46 \mathrm{Sec}$ & $357.69 \mathrm{Sec}$ & $429.37 \mathrm{Sec}$ & $376.08 \mathrm{Sec}$ \\
\hline \multirow[t]{4}{*}{ Man-Made Centroid } & $308.55 \mathrm{Sec}$ & $335.20 \mathrm{Sec}$ & 416.16 Sec & $369.42 \mathrm{Sec}$ \\
\hline & & $\mathrm{Vm} \mathrm{m}_{1}$ & $\mathrm{Vm \# 2}$ & $\mathrm{Vm} \# 4$ \\
\hline & & \multicolumn{3}{|c|}{ Hyper-V } \\
\hline & & 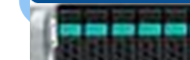 & & 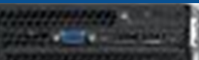 \\
\hline
\end{tabular}

Fig. 11. Results of the experiment in four virtual machines cloud environment. 


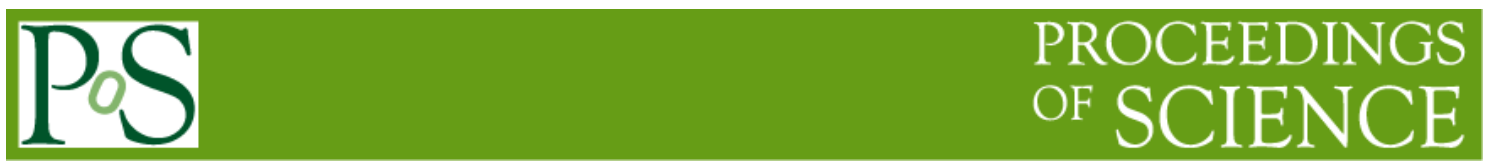

\begin{tabular}{|c|c|c|c|c|}
\hline \multicolumn{3}{|c|}{$K=5$} & \multicolumn{2}{|c|}{$K=10$} \\
\hline \multicolumn{5}{|c|}{ Original Data $(11,197,444$ points $)$} \\
\hline Average time $($ Run $=10)$ & Map $=1$ & Map $=12$ & Map=1 & Мap $=12$ \\
\hline Random Centroid & $315.28 \mathrm{Sec}$ & $672.20 \mathrm{Sec}$ & $348.97 \mathrm{Sec}$ & $740.38 \mathrm{Sec}$ \\
\hline Man-Made Centroid & $284.44 \mathrm{Sec}$ & $567.59 \mathrm{Sec}$ & $333.82 \mathrm{Sec}$ & $571.01 \mathrm{Sec}$ \\
\hline \multicolumn{5}{|c|}{ Reduced Data (165,707 points) } \\
\hline & Map $=1$ & Map $=2$ & Map=1 & Map $=2$ \\
\hline Random Centroid & $282.59 \mathrm{Sec}$ & $277.58 \mathrm{Sec}$ & $297.29 \mathrm{Sec}$ & $285.59 \mathrm{Sec}$ \\
\hline Man-Made Centroid & $258.64 \mathrm{Sec}$ & $252.42 \mathrm{Sec}$ & $288.69 \mathrm{Sec}$ & $279.01 \mathrm{Sec}$ \\
\hline
\end{tabular}

Fig. 12. Results of the experiment in three machines cloud environment.

From Fig. 11 and Fig. 12, we can readily find out three facts: (1) man-made centroid approach is better than random centroid in two kinds of cloud environments; (2) computation time of $K=10$ is more than $K=5$ due to complexity; (3) and more Maps can result less computation time in Random Centroid case.

\section{Conclusion}

By novel $K$-means approach, users can choose $K$ initial centroids by themself on tablet computer; app transmits these elements to cloud platform; and back-end system handles images processing immediately. In future research, we will focus on the accuracy of the classifications (automatic determination of the number of clusters), and propose a new approach to generate initial centroid automatically to accelerate the convergence time of algorithm.

Acknowledgment

This work is supported by the National Science Council of Taiwan, under grant NSC100-2625-M-035-003, NSC100-2119-M-035-001, NSC101-2625-M-035-002, and NSC101-2119-M-035-003. 


\section{References}

[1] J. B. MacQueen, "Some Methods for classification and Analysis of Multivariate Observations", Proceedings of 5-th Berkeley Symposium on Mathematical Statistics and Probability, Berkeley, University of California Press, 1:281-297, 1967.

[2] B. Furht and A. Escalante, "Handbook of Cloud Computing", Springer, 2011.

[3] L. George, "HBase: The Definitive Guide", O'Reilly Media, 2011.

[4] T. White, "Hadoop: The Definitive Guide, 3rd Edition", O'Reilly Media, 2012.

[5] F. C. Lin, L. K. Chung, W. Y. Ku, L. R. Chu, T. Y. Chou, The Framework of Cloud Computing Platform for Massive Remote Sensing Images, IEEE AINA 2013. (Accepted) 